\title{
Wolbachia endobacteria depletion by doxycycline as antifilarial therapy has macrofilaricidal activity in onchocerciasis: a randomized placebo-controlled study
}

\author{
Achim Hoerauf $\cdot$ Sabine Specht $\cdot$ Marcelle Büttner $\cdot$ Kenneth Pfarr $\cdot$ Sabine Mand $\cdot$ Rolf Fimmers \\ Yeboah Marfo-Debrekyei • Peter Konadu • Alexander Yaw Debrah · Claudio Bandi • Norbert Brattig • \\ Anna Albers · John Larbi $\cdot$ Linda Batsa $\cdot$ Mark J. Taylor · Ohene Adjei · Dietrich W. Büttner
}

Published online: 26 January 2008

(c) Springer-Verlag 2008

\section{Erratum to: Med Microbiol Immunol} DOI 10.1007/s00430-007-0062-1

The HTML version of this article unfortunately contained a mistake.

Mark J. Taylor was not listed among the authors.

The online version of the original article can be found under doi:10.1007/s00430-007-0062-1.

\footnotetext{
A. Hoerauf $(\bowtie) \cdot$ S. Specht $\cdot$ K. Pfarr $\cdot$ S. Mand

A. Y. Debrah · A. Albers Institute for Medical Microbiology, Immunology and Parasitology, University Clinic, Bonn, Sigmund-Freud-Str. 25, 53105 Bonn, Germany

e-mail: hoerauf@parasit.meb.uni-bonn.de

M. Büttner $\cdot$ N. Brattig $\cdot$ D. W. Büttner

Bernhard Nocht Institute for Tropical Medicine,

20359 Hamburg, Germany

R. Fimmers

Institute for Medical Biometry, Informatics, and Epidemiology,

University Clinic, Bonn, 53105 Bonn, Germany

Y. Marfo-Debrekyei · A. Y. Debrah · J. Larbi · L. Batsa · O. Adjei

Kumasi Centre of Collaborative Research,

Kumasi, Ghana
}

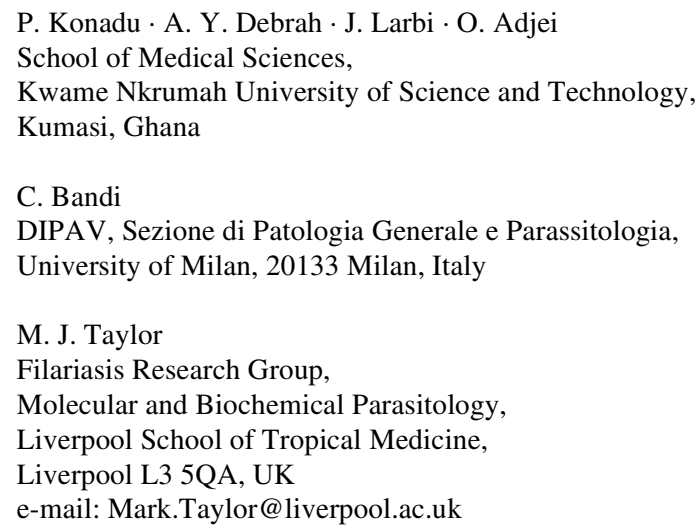

